\title{
Ferromagnetic Nanostructures Incorporated in Quasi-One-Dimensional Porous Silicon Channels Suitable for Magnetic Sensor Applications
}

\author{
P. Granitzer, K. Rumpf, and H. Krenn \\ Institute of Physics, University of Graz, Universitätsplatz 5, 8010 Graz, Austria
}

Received 15 March 2006; Revised 18 July 2006; Accepted 19 September 2006

Mesoporous silicon structures are fabricated during an anodization process of highly doped n-type silicon in hydrofluoric acid solution. The resulting pores are oriented perpendicular to the surface and exhibit a diameter of about $50 \mathrm{~nm}$ and a length up to $50 \mu \mathrm{m}$, controlled by the etching time. The growth of the pores is self-organized and depends on the crystal orientation of the used silicon wafer. The achieved channels, highly oriented along the (100) direction, are filled with nickel in a second electrochemical step. The deposition process leads to a distribution between high aspect ratio Ni-wires and Ni-particles of the incorporated metal. This achieved (porous silicon/Ni)-nanocomposite system exhibits a twofold switching behavior of the magnetization curve at two different field ranges. This property gives rise to high-magnetic field sensor applications based on a silicon technology.

Copyright (C) 2006 P. Granitzer et al. This is an open access article distributed under the Creative Commons Attribution License, which permits unrestricted use, distribution, and reproduction in any medium, provided the original work is properly cited.

\section{INTRODUCTION}

There is a great demand of nanostructures, especially nanowires in a nonmagnetic matrix [1] for a lot of promising applications in today's data storage technology, spintronic as well as sensor applications. The most popular are porous alumina templates, growing in a hexagonal honeycomb-like structure which is intensively investigated $[2,3]$. These structures are filled with a ferromagnetic material like $\mathrm{Ni}$ and the achieved magnetic properties are examined [4-6] up to now in the low-field regime below $1 \mathrm{~T}$ only. The introduced nanocomposite system, consisting of Ni-structures embedded in a porous silicon skeleton with high-aspect ratio channels (> 300), exhibits a feature at high-magnetic fields between $3 \mathrm{~T}$ and $6 \mathrm{~T}$, which leads to a possible application of a high-magnetic field sensor of high sensitivity. This magnetic high-field behavior depends on the temperature as well as the electrochemical loading conditions. Furthermore this deposition technique of metallic $\mathrm{Ni}$ in silicon is inexpensive and requires only two electrochemical steps in comparison to the multistep process of anodic oxidation of aluminium with subsequent metallization. The structure of this nanocomposite system is visualized by SEM. The verification of nickel within the pores is carried out by AES [7] and EDX spectroscopy. Magnetic measurements are performed by a SQUID magnetometer in the field range $\pm 7 \mathrm{~T}$.

\section{EXPERIMENTAL DETAILS}

Porous silicon (PS) generally shows an irregular growth of a sponge-like structure with interconnected channels in the diameter regime between $2 \mathrm{~nm}$ and $4 \mathrm{~nm}$. Mesopores with pores between $5 \mathrm{~nm}$ and $50 \mathrm{~nm}$ grow in a dendritic manner with a star-like cross-section of the pores. In this work we present a mesoporous silicon structure with highly oriented pores and dendrites smaller than the porediameter, indicating that the channels are really separated from each other. A further remarkable feature is the sharp termination between porous layer and bulk silicon (see Figure 1) which is sufficient for light reflection in the mid-infrared range and gives rise to optical and magneto-optical applications after the channels are filled with a ferromagnetic material. Despite a whole pore length of about $30 \mu \mathrm{m}$, the corrugation of the pore tips at this border lies in the range of about $10 \mathrm{~nm}$.

The mesoporous silicon layer is fabricated during anodization in a hydrofluoric acid solution. The essential parameters to achieve oriented channels with a diameter of a few tens of nanometers are the current density $\left(100 \mathrm{~mA} / \mathrm{cm}^{2}\right)$, 


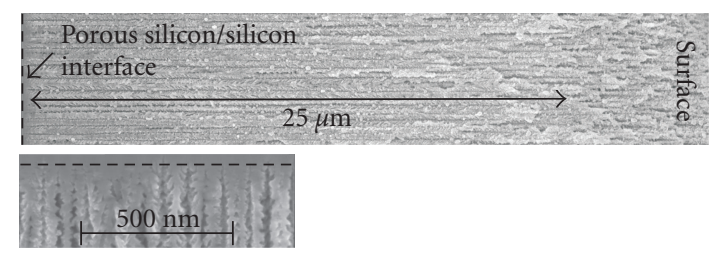

FIGURE 1: Scanning electron micrograph-with a magnification of 30000 - of a cleaved edge of a sample show the pores in full length as well as the sharp termination between porous layer and bulk silicon. The pores are oriented strictly perpendicular to the surface. The small image below indicates that the roughness of the porous silicon/bulk silicon interface lies in the range of $10 \mathrm{~nm}$.

electrolyte concentration ( $10 \mathrm{wt} \% \mathrm{HF}$ ), as well as the doping concentration of the used wafer $\left(10^{18} \mathrm{~cm}^{-3}\right)$. These parameters have to match accurately to obtain straight mesopores oriented perpendicular to the surface. Furthermore the bath temperature is kept at $20^{\circ} \mathrm{C}$ and the etching time is varied within the range of a few minutes to achieve a porous layer between $10 \mu \mathrm{m}$ and $50 \mu \mathrm{m}$ since the etching rate is about $5 \mu \mathrm{m} / \mathrm{min}$. To obtain a flat surface without cracks, a post treatment of the sample is necessary, like supercritical drying [8] or replacing of water by a liquid of lower surface tension.

The filling procedure of the channels with $\mathrm{Ni}$ is carried out in a galvanic process using nickel chlorine $\left(\mathrm{NiCl}_{2}\right)$ and boric acid $\left(\mathrm{H}_{3} \mathrm{BO}_{3}\right)$ as electrolyte. The deposition is performed in a pulsed way to get a homogeneous exchange of the electrolyte along the entire pore length (after [9] with modified electrolyte and deposition conditions). Otherwise, if there is a gradient of electrolyte concentration along the pores, they are blocked and not completely filled with nickel. However, the parameters of the electrochemical filling procedure can be varied to achieve different Ni-distributions within the samples. Typical used electrolyte concentrations are $150-160 \mathrm{~g} / \mathrm{l} \mathrm{NiCl}{ }_{2}$ and $50-55 \mathrm{~g} / \mathrm{l} \mathrm{H}_{3} \mathrm{BO}_{3}$. The current density (j) is varied between 20 and $50 \mathrm{~mA} / \mathrm{cm}^{2}$, the pulse duration $\left(t_{P}\right)$ ranges within a few tens of seconds. Ni is either concentrated near the surface region of the sample or at the pore tips or it is homogeneously spread over the whole pore length, as demonstrated in the EDX images in Figure 2. The EDX images are performed at $3 \mathrm{keV}$ showing qualitative elemental maps to get a rough survey of the Ni-distribution at the cleaved edge of the specimens. In these pictures the brightness of each pixel is controlled by the intensity of the chosen X-ray line at the spot, and higher brightness corresponds to a higher element concentration.

\section{STRUCTURAL AND MAGNETIC CHARACTERIZATIONS}

Considering the top view of the surface of the fabricated mesoporous template, the pores show a self-organization in a quadratic-like way with a quite homogeneous distribution of the pore diameter (Figure 4) which is mainly influenced by the (100) crystal orientation of the used silicon wafer. Scanning electron microscopy (SEM) with subsequent image processing demonstrates the square-like arrangement of the etched channels in Figure 3.

The electrodeposited Ni-structures within the pores consist of high-aspect ratio $\mathrm{Ni}$-wires as well as Ni-particles, indicated by structural as well as magnetic characterizations. Magnetic measurements are performed by a SQUID magnetometer (MPMS XL, Quantum Design) in the field range between $\pm 7 \mathrm{~T}$ and a temperature range from $4.2 \mathrm{~K}$ up to $300 \mathrm{~K}$. The magnetization curve shows two switching fields at different magnetic field ranges: one at low-magnetic fields around 500 Oe (HSW1) and the other at higher fields (HSW2) in the range of a few tesla. Ferromagnetic particles contributing to the magnetization signals which are below $200 \mathrm{~nm}$ in size are single domain [10]. An example of two $\mathrm{Ni}$-particles of about $150 \mathrm{~nm}$ and $50 \mathrm{~nm}$ is shown in Figure 5 by EDX images.

The hystereses loops in this low-field regime correlate with the size and amount of ferromagnetic particles. The coercivity for a magnetic field perpendicular to the sample surface ranges between $200 \mathrm{Oe}$ and $600 \mathrm{Oe}$, depending on the Ni-loading of the sample. If the magnetic field is applied in-plane, the coercivity decreases and shows values between 90 Oe and $150 \mathrm{Oe}$. The squareness $M_{R} / M_{s}$ varies between $20 \%$ and $70 \%$, depending on the magnetic field if applied out-of-plane or in-plane, respectively. Note that the coercivity shows a 30 -fold increase with respect to bulk $\mathrm{Ni}$, having a coercivity of $20 \mathrm{Oe}$, mainly caused by domain wall motion. With increasing temperature, the coercivity decreases as listed in Table 1. The presented system shows an analogous magnetic behavior in this low-field region as porous anodic alumina templates are filled with a ferromagnetic metal [1113].

In using the following relation [14]:

$$
r_{c}=\frac{36 \cdot \sqrt{A K_{1}}}{\mu_{0} M_{s}^{2}}
$$

the critical radius of Ni single-domain particles results in $r_{c} \approx 50 \mathrm{~nm}$. Most of the particles found in the sample are below $50 \mathrm{~nm}$ in diameter and can therefore be assumed as single domain. Due to the spheroidal-like shape, the easy axis is isotropic without any preferential direction, except for the small crystalline anisotropy of $\mathrm{Ni}$ which is negligible due to the polycrystalline structure of the wires. In this low-field range, the saturation magnetization is nearly equal for magnetic field direction parallel or perpendicular to the surface, respectively. For particles with a radius greater than the coherence radius $\mathrm{R}_{\text {coh }}(\sim 20 \mathrm{~nm}$ for $\mathrm{Ni}[15])$, magnetization reversal occurs favourably in the curling mode [16-18]. Calculating the coercive field of a particle with a diameter of $60 \mathrm{~nm}$ by using the following relation, [17, equation (2)], for the curling mode, we achieve a coercivity of about 450 Oe for a sphere, which is in good agreement with the measurements

$$
H_{C}=\frac{2 K_{1}}{\mu_{0} M_{s}}+\frac{c(N) A}{\mu_{0} M_{s} r^{2}}
$$

$K_{1}$ magnetocrystalline anisotropy, $M_{s}$ saturation magnetization, $N$ demagnetizing factor, $A$ exchange stiffness constant, $c$ geometrical factor depending on the aspect ratio. 


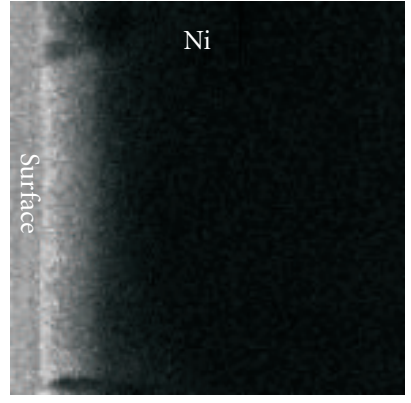

(a)

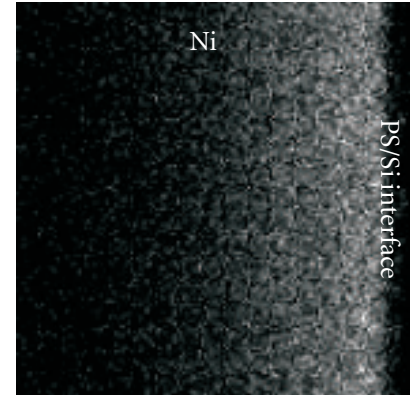

(b)

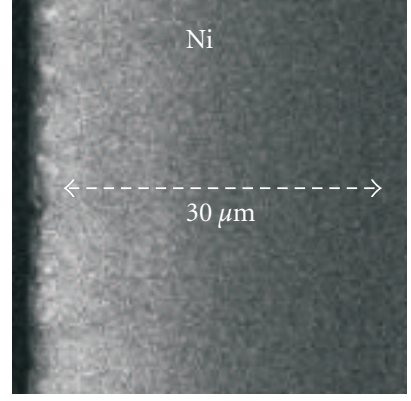

(c)

Figure 2: EDX scans at $3 \mathrm{keV}$ show different Ni-distributions controlled by changed loading conditions. Areas of higher brightness mean higher Ni-concentration. (a) Shows an accumulation of Ni near the surface $\left(j=10 \mathrm{~mA} / \mathrm{cm}^{2}, t_{P}=20\right.$ seconds), (b) indicates that the nucleation of $\mathrm{Ni}$ starts at the pore bottom $\left(\mathrm{j}=50 \mathrm{~mA} / \mathrm{cm}^{2}, t_{P}=10\right.$ seconds $)$ and $(\mathrm{c})$ shows a nearly homogeneous Ni-distribution $(\mathrm{j}=$ $20 \mathrm{~mA} / \mathrm{cm}^{2}, t_{P}=20$ seconds) within the porous layer.

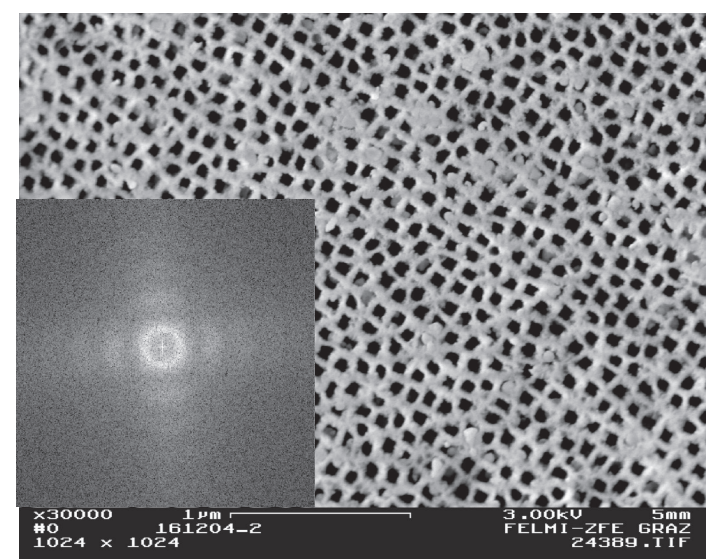

FIGURE 3: Scanning electron micrograph (SEM) of the top view of the surface of a porous silicon sample demonstrates the quadraticlike arrangement of the pore growth. In the inset, a corresponding 2D-Fourier transformed reciprocal image demonstrating the pattern of a square grid with a certain degree of disorder can be seen.

The nanocomposite system consists also of nanowires with a maximum diameter corresponding to the porediameter of the porous silicon template. The magnetization reversal of Ni-nanowires depends strongly on the wirediameter and magnetostatic interaction between the wires also influences the reversal mechanism [19-21]. Considering diameters greater than the coherence radius, the curling mode is dominant. Therefore the coercivity of such wires can be estimated by using (2) and is in the range of about $300 \mathrm{Oe}$, which is also in agreement with experimental data of the lowfield hysteresis loop.

This bimodal nanocomposite system shows a further characteristic switching field at higher magnetic fields of a few tesla presented in Figure 6. The mechanisms leading to this depression in the magnetization curve are not fully understood yet, but there are some remarkable points in considering the magnetization curve of Figure 6 . First it can be

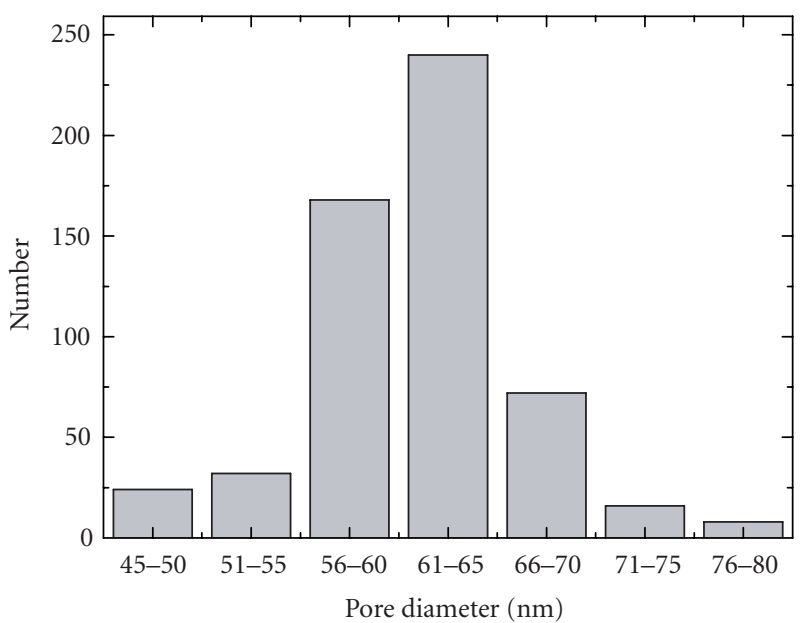

FIGURE 4: Histogram of a typical rather homogeneous distribution of the pore diameter within one porous silicon sample. The main pore diameter ranges between $56 \mathrm{~nm}$ and $70 \mathrm{~nm}$, considering a sample area of about $3 \mu \mathrm{m} \times 3 \mu \mathrm{m}$.

seen that the negative magnetization peak occurs at rather high fields between $4 \mathrm{~T}$ and $6 \mathrm{~T}$ (Figures 6 and 7) which is much higher than interwire dipolar coupling fields. A second point is the slight increase of the magnetization signal for fields greater than HSW1 up to field strengths at the beginning negative slope of the magnetization curve. Furthermore no hysteresis can be observed in the high-field range above HSW1. At last it can be seen that the described magnetic behavior at high fields can only be observed in an external magnetic field perpendicular to the sample surface (parallel to the nanowires) but does not occur if the magnetic field is applied parallel to the sample surface. Taking into account all these facts, we suggest the following as a first starting point for further investigations of the mentioned magnetic behavior. In order to comply all these experimental results, it is justified to assume transitions between antiparallel and parallel alignments of Ni-nanowires. The strong depression, 


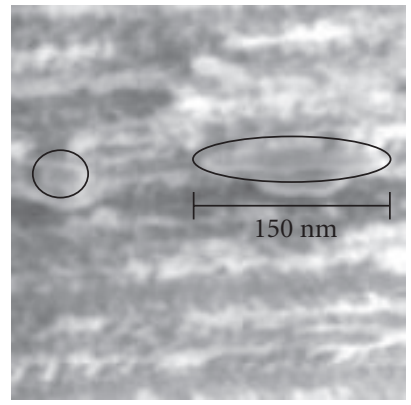

(a)

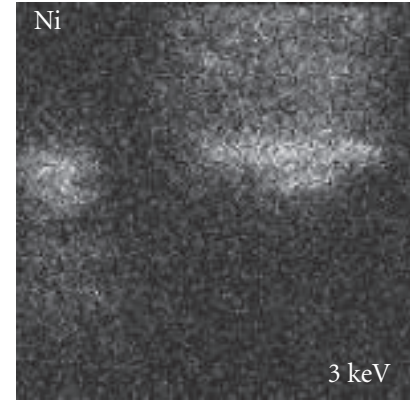

(b)

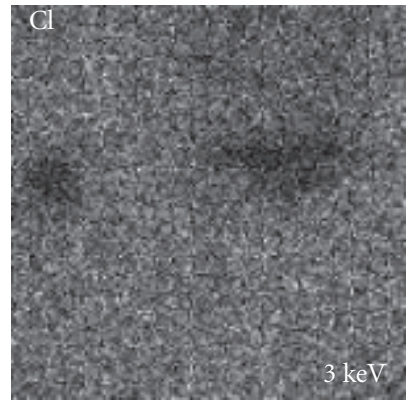

(c)

FIGURE 5: EDX scans showing a zoomed sample area containing two Ni-particles indicated by ellipses in picture (a) which is obtained from the backscattered electrons. (b) shows the Ni-distribution being confined to this two regions and (c) demonstrates the absence of chlorine in these two areas.

TABLE 1: Coercivity of a Ni-nanowire sample (pitch: $\sim 120 \mathrm{~nm}$ ) in dependence on the temperature when the magnetic field is applied parallel to the long wire axis. The coercive field decreases with increasing temperature.

\begin{tabular}{l|ccccc}
\hline $\mathbf{T}$ & 4.2 & 25 & 50 & 100 & 250 \\
\hline $\mathbf{H}_{\mathbf{C}}$ & 330 & 320 & 260 & 200 & 140 \\
\hline
\end{tabular}

which can be observed as descending magnetization in the hysteresis loop for an increasing magnetic field $(H>4 \mathrm{~T})$, could be understood by this increase of antiparallel alignment of single-domain wires $\left(H_{\mathrm{ex}}=\mathrm{HSW} 2\right)$. If the external field is further increased $\left(H_{\mathrm{ex}}>\mathrm{HSW} 2\right)$, finally all the wires order into the same direction and are aligned with the magnetic field. Hence, the system gets saturated above $6.5 \mathrm{~T}$ (Figure 6).

The main problem of this explanation is the interaction between the Ni-wires leading to an antiparallel alignment at rather high fields of a few tesla which is still under investigation and not completely understood yet. As mentioned before, the interaction mechanism cannot be of dipolar character because it is too weak to obtain switching fields at high fields of a few tesla. In order to elucidate the behavior with respect to all experimental results, an assumed exchange interaction has to fulfill some requirements. First, it has to take place at high-magnetic fields and as a second point it has to act across the distance between the wires. The latter point is critical due to the pitch of the porous silicon template in the range of tens of nanometers. This can be eased taking into account that the pores are not ideal cylindrical, exhibiting not completely smooth pore walls but dendritic growth. This affects an effective distance between the wires which is considerably smaller in the real system than in an array of ideal cylindrical wires. On behalf of this consideration, the effective distance is in the range of only a few nanometers and this is not too large for an exchange interaction to take place.

A further interesting behavior is the temperature dependence of the second switching field. HSW2 decreases with increasing temperature (Figure 7). Beneath $30 \mathrm{~K}$, the available magnetic field of $\pm 7 \mathrm{~T}$ in our SQUID magnetometer does not suffice to monitor a measurable depression of the

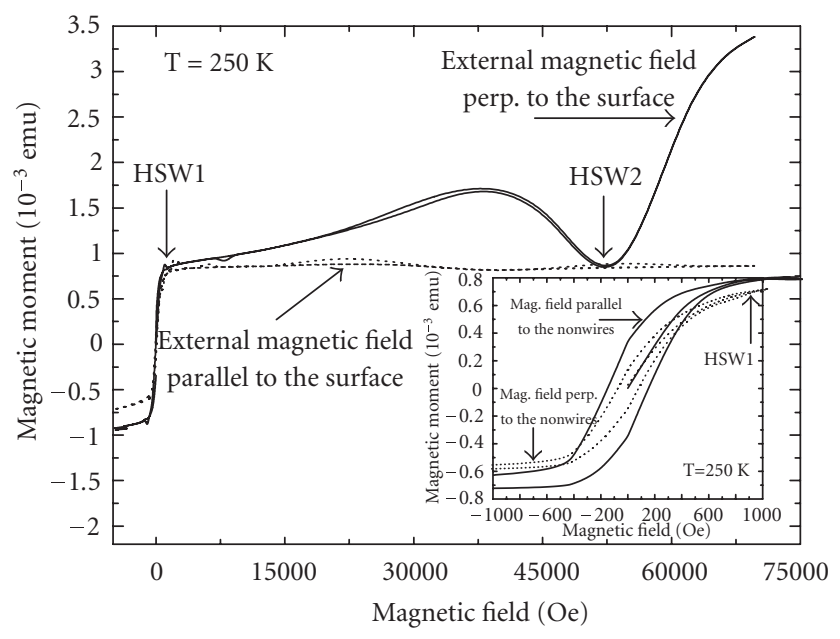

FIGURE 6: Magnetization curve showing the first switching field HSW1 at $500 \mathrm{Oe}$ (inset) as well as the second switching field HSW2 at $5.1 \mathrm{~T}$ which is suggested to be due to the mutual antiferromagnetic alignment of the Ni-wires. The diamagnetic contribution of the substrate is subtracted. If the applied magnetic field is parallel to the sample surface, no negative magnetization peak is observed.

magnetization signal. The temperature dependence of the second switching field is assumed to be due to the strong temperature dependence of the anisotropy constant $K_{1}$ of nickel, which generally decreases with increasing temperature [22].

The shape anisotropy constant is independent of the temperature and thus the uniaxial anisotropy constant $K_{1}$ remains as the only temperature-dependent entity. These observations correspond to experiments reported in the literature [20] where the nucleation fields also increase with decreasing temperature. Note, however, the one order of magnitude has smaller nucleation field than in our Ni/porous silicon samples. Usually the magnetocrystalline contributions tend to average out due to random distributions of the Ni-structures incorporated into the pores [23]. A further contribution is the magnetoelastic anisotropy caused 


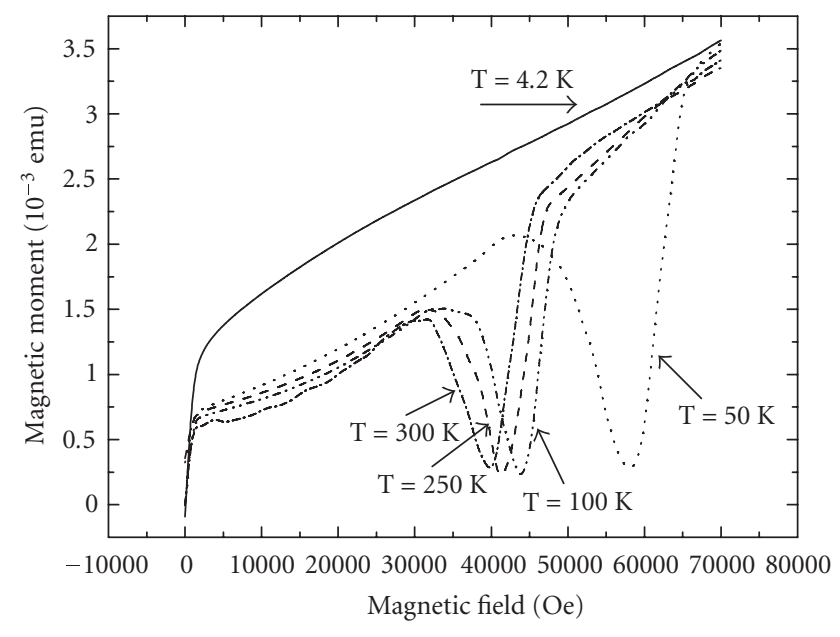

FIgURE 7: Temperature variation between $300 \mathrm{~K}$ and $4.2 \mathrm{~K}$ shifts the second switching field HSW2 from 3.9 T up to $5.7 \mathrm{~T}$.

by stress due to the lattice mismatch between the incorporated metallic $\mathrm{Ni}$ and $\mathrm{PS}$. These effects are mainly caused by the deposition conditions due to the bridging of the NiPS [24]. Furthermore the temperature-dependent behavior is also caused by the strain, due to the mismatch of the thermal expansion coefficients between nickel $\left(\alpha_{\mathrm{th}, \mathrm{Ni}}=13.4\right.$. $\left.10^{-6} \mathrm{~K}^{-1}\right)$ and porous silicon $\left(\alpha_{\mathrm{th}, \mathrm{PS}}=1.2 \cdot 10^{-6} \mathrm{~K}^{-1}\right)$. The magnetoelastic anisotropy $K_{\mathrm{el}}$ [25] decreases with increasing temperature from $K_{\mathrm{el}}=-0.5 \cdot 10^{6} \mathrm{Jm}^{-3}$ to $K_{\mathrm{el}}=-3.39$. $10^{6} \mathrm{Jm}^{-3}$ taking into account that the strain of $\mathrm{Ni}$ has a value of $\varepsilon \sim-3.5 \cdot 10^{-3}$ at $4.2 \mathrm{~K}$ and $\varepsilon \sim-5.246 \cdot 10^{-4}$ at $250 \mathrm{~K}$. A further correlation is found between the second switching field and the loading condition of the Ni-filling process of the channels. Varying the electrochemical parameters like current density and pulse duration leads to a different distribution of particles and wires and therefore to various coercivities as well as negative magnetization peaks. The Nicontent varies with the loading conditions. For $H_{C}=350 \mathrm{Oe}$, a Ni-content of $3.813 \cdot 10^{-6} \mathrm{~cm}^{3}$ is estimated and it decreases with decrescent $H_{C}=220 \mathrm{Oe}$, Ni-content $=1.9 \cdot 10^{-6} \mathrm{~cm}^{3}$. Figure 8 demonstrates that also the depression in the highfield region can be influenced by different deposition conditions.

The switching in the range of a few tesla works in a broad temperature range even up to room temperature, which is of great interest for technical applications. A shift of the depression of the magnetization curve to higher magnetic fields is observable if the temperature decreases (see Figure 7). The steep decrease of the $\mathrm{M}(\mathrm{H})$ curves around the second switching field followed by a steep increase gives rise to applications in magnetic sensor technology. Furthermore, the high-field switching feature can be controlled by different Ni-loading conditions. Changing the parameters of the electrochemical deposition alters the distribution of Ni-particles and $\mathrm{Ni}$-wires, leading to different slopes of the declining and inclining branches of the magnetization curve (see Figure 8). Higher steepness of the magnetization curve around

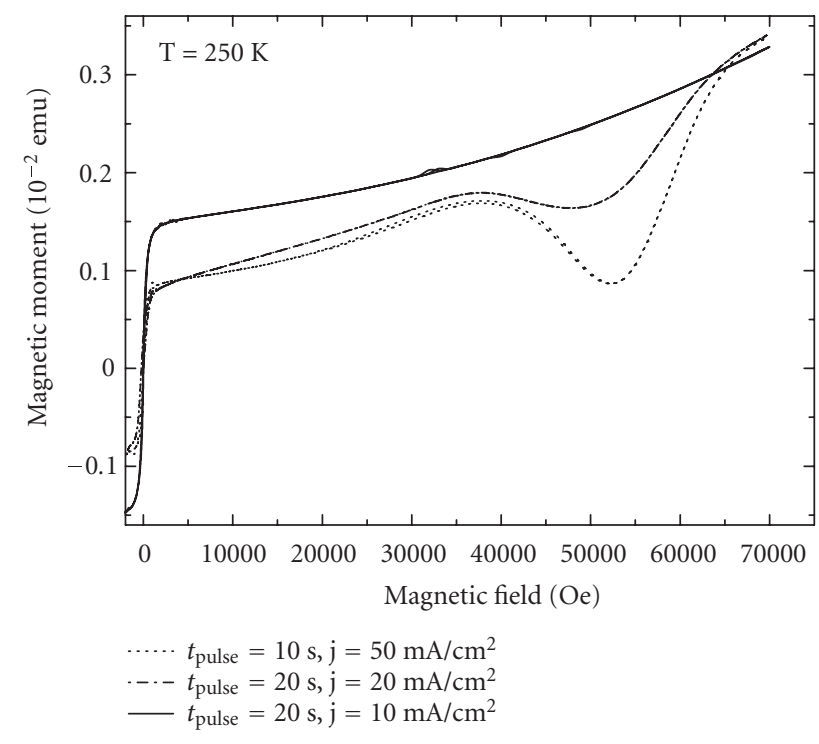

FIGURE 8: Onset of a negative magnetization at the second switching field for different Ni-loading conditions, of a (Ni/porous silicon)-nanocomposite. The corresponding EDX images of the Nidistributions are shown in Figure 2.

the second switching field means higher sensitivity for a magnetic field sensor. The width of the switching peak is responsible for the field range within the sensor working. However, the strong correlation of the incorporated nanostructures and of the temperature with the switching field has been demonstrated in this work.

Because of the temperature-dependent shift of the switching field, a magnetic field, sensor is possible to be realized with both, high sensitivity as well as a wide working range between $3 \mathrm{~T}$ and $6 \mathrm{~T}$. For applications at fixed temperatures (i.e., room temperature), the sensor specification can only be influenced by the amount of electrochemical Nideposition. In this case, the sensitivity and the working range are competing features. In consideration of this fact, special nanocomposite systems can be tailored, to customize a favored magnetization curve. For example, a sharp and narrow peak of the $\mathrm{M}(\mathrm{H})$ curve is realizable. A specimen of such a behavior is a very promising candidate for a high-magnetic field sensor with a small working range but with a rather high sensitivity, and can be used to stabilize a magnetic field at a fixed temperature.

\section{CONCLUSIONS}

The proposed method to fabricate Ni-nanowires embedded in a porous silicon matrix is a two-step electrochemical process. This procedure, managed by less steps, is inexpensive and opens a lot of opportunities for technical applications in silicon technology. In the first step, the porous silicon template is fabricated during an anodization process, using aqueous hydrofluoric acid as solution. The achieved porous structure consists of highly oriented channels with an extremely 
high-aspect ratio up to $1: 1000$. In a second step, this template is filled with Ni during pulsed electrodeposition. The obtained nanocomposite system consists of a distribution of particles and wires dependent on the electrochemical parameters of the deposition process like current density and pulse duration. The electrolyte concentration is also an important Ni-loading parameter. The structural characterization of this nanoscopic arrangement is carried out by scanning electron microscopy (SEM) and energy dispersive X-ray spectroscopy (EDXS). SEM investigations demonstrate that the pores are highly oriented perpendicular to the sample surface and that the pores are arranged in a square-like manner, shown by image processing of top view SEM images. EDXS indicates that the pores are filled with Ni down to the pore tips. With EDXS the distribution of different elements within one sample is shown as well as the $\mathrm{Ni}$-distribution of differently processed samples.

The bimodal ferromagnetic nanocomposite consisting of $\mathrm{Ni}$-particles and Ni-wires shows an interesting twofold magnetic switching behavior at different external magnetic fields. The first switching in the low-field regime shows a clear anisotropic behavior of the hysteresis loops between in-plane and out-of-plane magnetizations. This behavior is similar to that one known from other nanowire arrays like in porous anodic alumina templates. Due to the geometry of the system, the magnetization reversal process occurs mainly by curling. The mechanisms of the second switching at high fields cannot be explained completely up to now but are still under investigation.

This (porous silicon/Ni)-nanocomposite exhibiting the described novel bimodal magnetic behavior is a promising candidate for applications in magnetic high-field sensor devices. An advantage of this system is the use of silicon as basic material which can be integrated in today's semiconductor microtechnology.

\section{ACKNOWLEDGMENTS}

The authors thank Dr. P. Pölt and Dr. A. Reichmann from the Institute for Electron Microscopy (FELMI) at the University of Technology in Graz for SEM and EDX and the Austrian FWF-fund for its Grant under Project P15397.

\section{REFERENCES}

[1] T. M. Whitney, J. S. Jiang, P. C. Searson, and C. L. Chien, "Fabrication and magnetic properties of arrays of metallic nanowires," Science, vol. 261, no. 5126, pp. 1316-1319, 1993.

[2] O. Jessensky, F. Müller, and U. Gösele, "Self-organized formation of hexagonal pore arrays in anodic alumina," Applied Physics Letters, vol. 72, no. 10, pp. 1173-1175, 1998.

[3] H. Masuda and K. Fukuda, "Ordered metal nanohole arrays made by a two-step replication of honeycomb structures of anodic alumina," Science, vol. 268, no. 5216, pp. 1466-1468, 1995.

[4] K. Nielsch, R. B. Wehrspohn, J. Barthel, et al., "Hexagonally ordered $100 \mathrm{~nm}$ period nickel nanowire arrays," Applied Physics Letters, vol. 79, no. 9, pp. 1360-1362, 2001.
[5] R. Skomski, H. Zeng, and D. J. Sellmyer, "Incoherent magnetization reversal in nanowires," Journal of Magnetism and Magnetic Materials, vol. 249, no. 1-2, pp. 175-180, 2002.

[6] L. Clime, P. Ciureanu, and A. Yelon, "Magnetostatic interactions in dense nanowire arrays," Journal of Magnetism and Magnetic Materials, vol. 297, no. 1, pp. 60-70, 2006.

[7] P. Granitzer, K. Rumpf, S. Surnev, and H. Krenn, "Squidmagnetometry on ferromagnetic Ni-nanowires embedded in oriented porous silicon channels," Journal of Magnetism and Magnetic Materials, vol. 290-291, part 1, pp. 735-737, 2005.

[8] L. T. Canham, A. G. Cullis, C. Pickering, O. D. Dosser, T. I. Cox, and T. P. Lynch, "Luminescent anodized silicon aerocrystal networks prepared by supercritical drying," $\mathrm{Na}$ ture, vol. 368, no. 6467, pp. 133-135, 1994.

[9] K. Nielsch, F. Müller, A.-P. Li, and U. Gösele, "Uniform nickel deposition into ordered alumina pores by pulsed electrodeposition," Advanced Materials, vol. 12, no. 8, pp. 582-586, 2000.

[10] J. I. Martín, J. Nogués, K. Liu, J. L. Vicent, and I. K. Schuller, "Ordered magnetic nanostructures: fabrication and properties," Journal of Magnetism and Magnetic Materials, vol. 256, no. 1-3, pp. 449-501, 2003.

[11] D.-H. Qin, H.-L. Zhang, C.-L. Xu, T. Xu, and H.-L. Li, "Magnetic domain structure in small diameter magnetic nanowire arrays," Applied Surface Science, vol. 239, no. 3-4, pp. 279-284, 2005.

[12] M. Vázquez, K. Pirota, J. Torrejón, D. Navas, and M. Hernández-Vélez, "Magnetic behaviour of densely packed hexagonal arrays of $\mathrm{Ni}$ nanowires: influence of geometric characteristics," Journal of Magnetism and Magnetic Materials, vol. 294, no. 2, pp. 174-181, 2005.

[13] M. Knobel, L. C. Sampaio, E. H. C. P. Sinnecker, P. Vargas, and D. Altbir, "Dipolar magnetic interactions among magnetic microwires," Journal of Magnetism and Magnetic Materials, vol. 249, no. 1-2, pp. 60-72, 2002.

[14] D. J. Sellmyer, M. Zheng, and R. Skomski, "Magnetism of FE, CO and NI nanowires in self-assembled arrays," Journal of Physics: Condensed Matter, vol. 13, no. 25, pp. R433-R460, 2001.

[15] A. Aharoni, "Incoherent magnetization reversals in elongated particles," Journal of Magnetism and Magnetic Materials, vol. 196-197, pp. 786-790, 1999.

[16] C. A. Ross, R. Chantrell, M. Hwang, et al., "Incoherent magnetization reversal in 30-nm Ni particles," Physical Review B: Condensed Matter and Materials Physics, vol. 62, no. 21, pp. 14252-14258, 2000.

[17] R. Skomski, "Nanomagnetics," Journal of Physics: Condensed Matter, vol. 15, no. 20, pp. R841-R896, 2003.

[18] R. Hertel and J. Kirschner, "Magnetization reversal dynamics in nickel nanowires," Physica B: Condensed Matter, vol. 343, no. 1-4, pp. 206-210, 2004.

[19] R. Wieser, U. Nowak, and K. D. Usadel, "Domain wall mobility in nanowires: transverse versus vortex walls," Physical Review B: Condensed Matter and Materials Physics, vol. 69, no. 6, Article ID 064401, 5 pages, 2004.

[20] S. Pignard, G. Goglio, A. Radulescu, et al., "Study of the magnetization reversal in individual nickel nanowires," Journal of Applied Physics, vol. 87, no. 2, pp. 824-829, 2000.

[21] R. Hertel, "Computational micromagnetism of magnetization processes in nickel nanowires," Journal of Magnetism and Magnetic Materials, vol. 249, no. 1-2, pp. 251-256, 2002.

[22] B. D. Cullity, Introduction to Magnetic Materials, Addison Wesley, Reading, Mass, USA, 1972. 
[23] H. Zeng, S. Michalski, R. D. Kirby, D. J. Sellmyer, L. Menon, and S. Bandyopadhyay, "Effects of surface morphology on magnetic properties of $\mathrm{Ni}$ nanowire arrays in self-ordered porous alumina," Journal of Physics: Condensed Matter, vol. 14, no. 4, pp. 715-721, 2002.

[24] H. Zeng, R. Skomski, L. Menon, Y. Liu, S. Bandyopadhyay, and D. J. Sellmyer, "Structure and magnetic properties of ferromagnetic nanowires in self-assembled arrays," Physical Review B: Condensed Matter and Materials Physics, vol. 65, no. 13, Article ID 134426, 8 pages, 2002.

[25] G. Bertotti, Hysteresis in Magnetism, Academic Press, New York, NY, USA, 1998. 

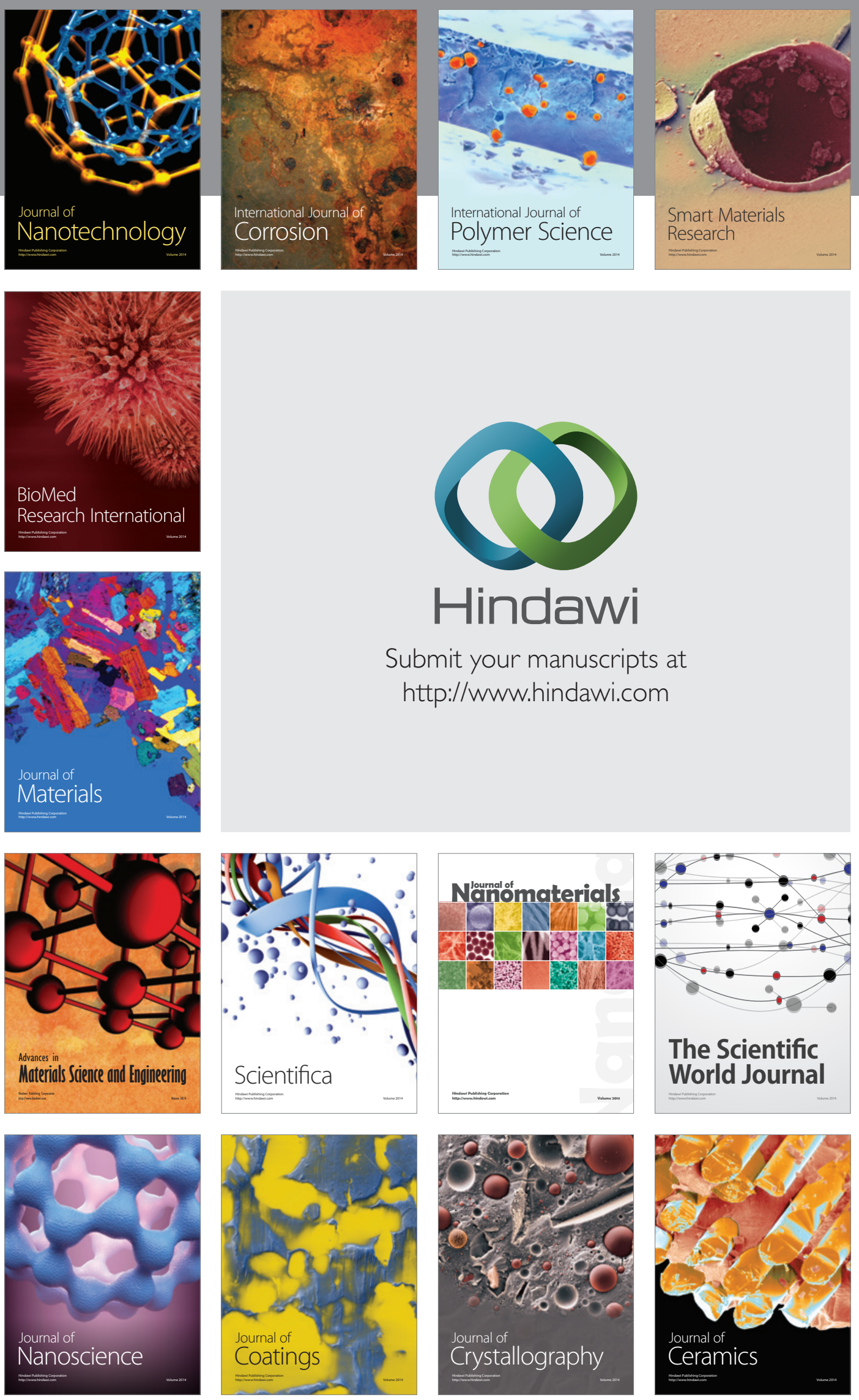

The Scientific World Journal

Submit your manuscripts at

http://www.hindawi.com

\section{World Journal}

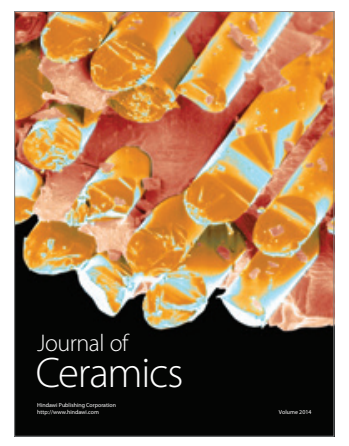

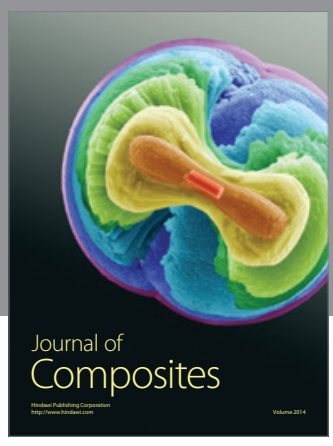
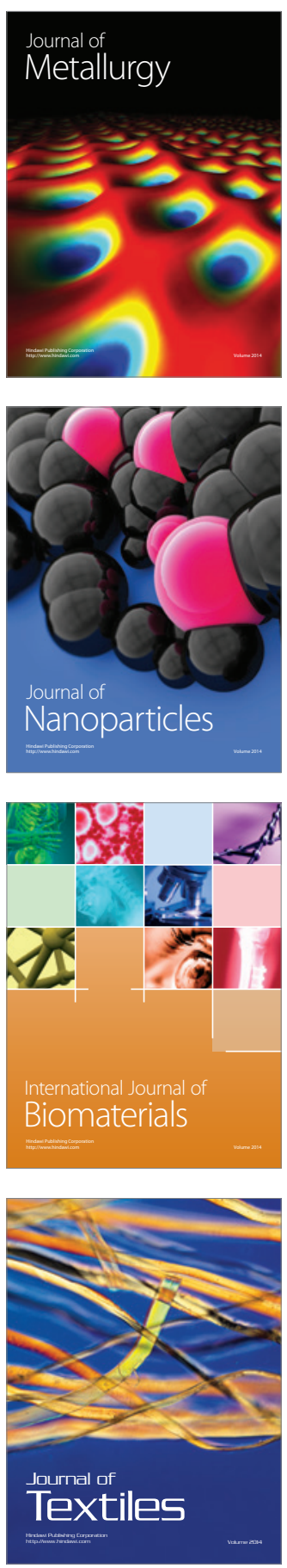